\title{
Spectrally resolved Motional Stark Effect measurements on ASDEX Upgrade
}

R. Reimer, ${ }^{1}$ A. Dinklage, ${ }^{1}$ R. Fischer ${ }^{2}{ }^{2}$ J. Hobirk, ${ }^{2}$ T. Löbhard, ${ }^{2}$ A. Mlynek, ${ }^{2}$ M. Reich, ${ }^{2}$ L. Sawyer, ${ }^{3}$ R. Wolf, ${ }^{1}$

and the ASDEX Upgrade 2 , a)

1) Max-Planck-Institut für Plasmaphysik, EURATOM Association, Teilinstitut Greifswald, Wendelsteinstraße 1, 17491 Greifswald, Germany

2) Max-Planck-Institut für Plasmaphysik, EURATOM Association, Boltzmannstraße 2, 85748 Garching, Germany

3) Department of Physics and Astronomy, University of Sheffield, Sheffield S3 $7 R H$, United Kingdom

(Dated: 8 January 2015)

A spectrally resolved MSE diagnostic has been installed at ASDEX Upgrade. The MSE data have been fitted by a forward model providing access to information about the magnetic field in the plasma interior. The forward model for the beam emission spectra comprises also the fast ion $\mathrm{D}_{\alpha}$ (FIDA) signal and the smearing on the CCD-chip. The calculated magnetic field data as well as the revealed (dia)magnetic effects are consistent with the results from equilibrium reconstruction solver. Measurements of the direction of the magnetic field are affected by unknown and varying polarization effects in the observation.

\section{INTRODUCTION}

The reconstruction of magnetic equilibria is crucial for the analysis of diagnostic results in magnetic confinement fusion experiments. In tokamaks, equilibrium calculations based on the solution of the Grad-Shafranov equation can be effectively constrained by measurements ${ }^{2}$. Examples for such experimental input for equilibrium reconstruction include magnetic flux measurements outside the plasma and Faraday rotation measurements ${ }^{3}$ yielding information from the plasma interior.

An active diagnostic measuring local components of the $\vec{B}$-field employs the Motional Stark Effect (MSE) of the emission from fast injected particles excited by the hot plasma $^{4,5}$. Due to the linear $\vec{B}$-field dependence of the Motional Stark-splitting, MSE measurements are routinely performed on the $\mathrm{D}_{\alpha}$-line of excited beam particles.

$\vec{E}=\vec{v}_{B} \times \vec{B}$ being the quantisation axis, the so-called $\sigma \pm(\Delta m= \pm 1)$ and $\pi(\Delta m=0)$ lines form the Stark multiplet $^{6,7} . \quad \pi$ and $\sigma$ lines differ in their polarization and their spectral splitting reflecting the Lorentz field strength. Quantitative spectroscopy of the entire Doppler-shifted spectrum from fast particles allows to determine of $|\vec{B}|$ from the Stark-splitting and, in principle, the direction of $\mathrm{B}$ from the ratio of $\sigma$ to $\pi$ components. This is because their emissions have varying characteristics $^{5}$. In contrast, Stark polarimetry ${ }^{4,8}$ on the unshifted $\sigma$ lines measures the orientation of the polarisation and is routinely used to estimate the pitch of the magnetic field in a fusion plasma ${ }^{4}$.

Spectral MSE (sMSE) measures the MSE spectrum to determine $\left|E_{L}\right|$ and thus $|\vec{B}|$ and retrieves information about $\vec{B} /|\vec{B}|$ from the $\sigma$ - and $\pi$ intensity ratios ${ }^{9}$. This paper describes the technical implementation of an sMSE

a) See authors list of A. Kallenbach et al., Nucl. Fusion 51(9), 094012 (2011). system on ASDEX Upgrade.

Experimentally the observed spectrum consists of the Stark multiplets and spurious components which have been identified to result from plasma edge emission, fast ions, charge exchange (CX) and impurity lines. The complexity of the spectrum requires detailed data analysis. Consequently, beyond the experimental implementation, particular emphasis was put on the modelling of the emission spectrum. The data analysis model and its impact on the interpretation of the data is the second aspect described in this paper. As a specific focus we address the capabilities of sMSE to assess diamagnetic effects.

\section{PHYSICS OF MSE}

The Stark-splitting and thus $|\vec{B}|$ can be determined by applying the Epstein-Schwarzschild formula:

$$
\Delta E_{n}(\mathrm{eV})=7.94198 \cdot 10^{-11} \times\left|\vec{E}_{L}\right|\left(\mathrm{Vm}^{-1}\right) n\left(n_{1}-n_{2}\right)
$$

where $n$ denotes the principal quantum number and $n_{1}$ and $n_{2}$ are parabolic quantum numbers ${ }^{10}$.

Contributions resulting from the Zeeman effect were neglected. This is reasonable for the $\mathrm{D}_{\alpha}$ beam emission with beam energies of the order of $30 \mathrm{keV} / \mathrm{amu}$ at the present geometry with a minimal angle between beam and magnetic field line of $\alpha_{\text {min }} \approx 55^{\circ}$. Under these conditions the relation between the energy splittings resulting from the Zeeman effect $\left(\gamma_{Z}\right)$ and resulting from the Stark effect $\left(\epsilon_{S t}\right)$ is:

$$
\begin{aligned}
\frac{\gamma_{Z}}{\epsilon_{S t}} & =\frac{\mu_{B}}{(3 / 2) a_{0} e} \cdot \frac{1}{v_{B} \sin \left(\alpha_{\min }\right)} \\
& =0.0036
\end{aligned}
$$

with the Bohr magneton, $\mu_{B}$, the Bohr radius, $a_{0}$, the elementary charge, $e$, and the beam velocity $v_{B}$.

The direction of the magnetic field is reflected by the Stark line ratio $T_{p}$ of the $\sigma$ and $\pi$ components due to 
their emission characteristics:

$$
\begin{aligned}
T_{p} & =\frac{\sum I_{\pi_{i}}}{\sum I_{\sigma_{i}}} \\
& \approx \frac{\sin ^{2} \theta}{1+\cos ^{2} \theta}
\end{aligned}
$$

with $\theta$ being the azimuth with respect to $\vec{E}^{11}$.

In order to take into account the non-statistical distributions of the Stark sub-levels a correction factor, $c_{n s}$, is introduced: $T_{p}^{n s}=T_{p} / c_{n s} . \quad c_{n s}$ depends mainly on the plasma density, magnetic field and injected beam energy $^{12}$.

\section{EXPERIMENTAL IMPLEMENTATION}

\section{A. Experimental set-up}

FIG. 1 shows the experimental set-up of the sMSE diagnostic at ASDEX Upgrade (major radius $R=1.65 \mathrm{~m}$, minor radius $r=0.5 \mathrm{~m})^{13,14}$. The maximum magnetic field is $B=3.1 \mathrm{~T}$. For the sMSE a magnetic field of about 2 to $2.6 \mathrm{~T}$ is applied. The beam emission comes from a $2.5 \mathrm{MW}$ heating beam. The beam, produced by a positive ion source injects neutral deuterium at energies of $60 \mathrm{keV}, 30 \mathrm{keV}$ and $20 \mathrm{keV}$ into the plasma and has a divergence of about $1^{\circ}$. At its vertical focal point (at $R=2.018 \mathrm{~m}$ ) the beam has a cross-section of $25 \mathrm{~cm} \times 27 \mathrm{~cm} \mathrm{width}^{15}$.

The $\mathrm{D}_{\alpha}$ emission, caused by the plasma beam interaction, can be observed by an array of $6 \times 10$ (vertical $\times$ horizontal) lines of sight (LOS) with varying observation angles. Thus, it is possible to detect signals along the beam axis at different radial positions. The sMSE diagnostic utilizes six of ten horizontal LOS.

For this prototypical study, the detection optics of the MSE polarimeter were shared. As shown in fig. 1, the LOSs intersect a mirror $(\mathrm{M})$, covered by a glass window (W1). Since the sMSE and the MSE polarimetry employ the same observation system, the beam emission signal also passes through the MSE polarimeter. A lens system (L1) collects the light to guide it through the components of the polarimeter (photo-elastic modulators, PEM1 and PEM2 and polariser, $\mathrm{P}$ ) in parallel rays.

For each LOS the collected light is relayed from the torus hall towards the diagnostic hall by a $50 \mathrm{~m}$ length optical fibre. A lens system (L2) is applied to focus the signal onto the fibres. Since the fibres (diameter $1 \mathrm{~mm}$ ) are arranged in one vertical line at the entrance of a Czerny-Turner spectrometer (focal length $0.75 \mathrm{~m}$, grating of $g=1200 \mathrm{~mm}^{-1}$ and F-number 6.5), a twodimensional frame transfer CCD $(1024 \times 1024$ with $13 \mu \mathrm{m}$ pixels) is used behind the exit slit to collect both the radial and spectral information from the beam emission. Since the signal was found to be affected by pixel smearing (cross-talk) effects, one channel is covered, (dark reference channel cf. fig. 1) to measure this effect. Moreover, one channel is used for wavelength referencing using the spectrum from a Neon spectral lamp. Experimentally a small spectral region of about $0.5 \mathrm{~nm}$ at $\lambda=656.1 \mathrm{~nm}$ is masked by a blocking wire at the exit slit of the spectrometer to suppress the signal resulting from cold $\mathrm{D}_{\alpha}$ edge emission line. In other case this intense emission line would lead to saturation on the CCD-chip. The intermediate image of the spectrum and blocking wire is projected onto the CCD-chip with a lens system (L3).

\section{B. Experimental results}

A typical spectrum and fitted data (as explained in section IV) for one position $(R=1.90 \mathrm{~m}, z=0.09 \mathrm{~m})$ at $t=2.37 \mathrm{~s}$ are displayed in the upper plot in fig. 2. The dominating feature is the CX emission line $\left(d_{\mathrm{CX}}\right)$ which is slightly shifted with respect to the suppressed cold $\mathrm{H}_{\alpha}$ and $\mathrm{D}_{\alpha}$ emission lines (at around $656.1 \mathrm{~nm}$ ). The area covered by the blocking wire is indicated by the gray rectangle in fig. 2. On the red-wing side impurity lines are observed at $657.8 \mathrm{~nm}$ and $658.2 \mathrm{~nm}$, e.g. CII $\left(d_{\mathrm{Imp}}\right)$.

On the blue-wing side $(653 \ldots 655 \mathrm{~nm})$ the MSE spectrum, consisting of three Stark multiplets (corresponding to the full, half and third beam energy) each Dopplershifted and overlapping is clearly visible. These are denoted $d_{\mathrm{MSE}}\left(\mathrm{E}_{0}\right), d_{\mathrm{MSE}}\left(\mathrm{E}_{1 / 2}\right), d_{\mathrm{MSE}}\left(\mathrm{E}_{1 / 3}\right)$. The fact that the MSE spectrum is overlapped partly by the CX emission line and completely by two flat and spectrally broad components (these being the FIDA emission line $d_{\text {FIDA }}$ and the cross-talk on the chip $d_{\mathrm{CT}}$ ) stresses the importance of a good description of these spectral features. The remarkably high quality of the fit is reflected in the lower plot by the goodness-of-fit. The error analysis, as well as the applied forward model will be outlined in detail in section IV.

\section{DATA ANALYSIS}

A typical approach to analyse the experimental data $(D)$ is to fit the modelled data $(d)$ to a multi-Gaussian model with a large set of fit parameters ${ }^{5}$. Here instead, the data are fitted to a forward model ${ }^{13,14}$. Practically, the forward model has much less parameters than the multi-Gaussian model. Furthermore, the forward model parameters are accessible to experimental error assessments and are easily employed for the application of constraints.

A flow-chart of the forward model is shown in fig. 3 . Based on the physical cause, namely the magnetic field, and the beam energy, as well as the beam observation geometry the signal detected on the CCD-chip is simulated. The magnetic field can be used as a constraint for equilibrium calculations ${ }^{16}$ (an example equilibrium, calculated with the CLISTE equilibrium solver, is given at the left hand side of the flow-chart). A typical sample of the detected experimental data is shown by the right plot of the flow-chart. The color coded signal of the beam 


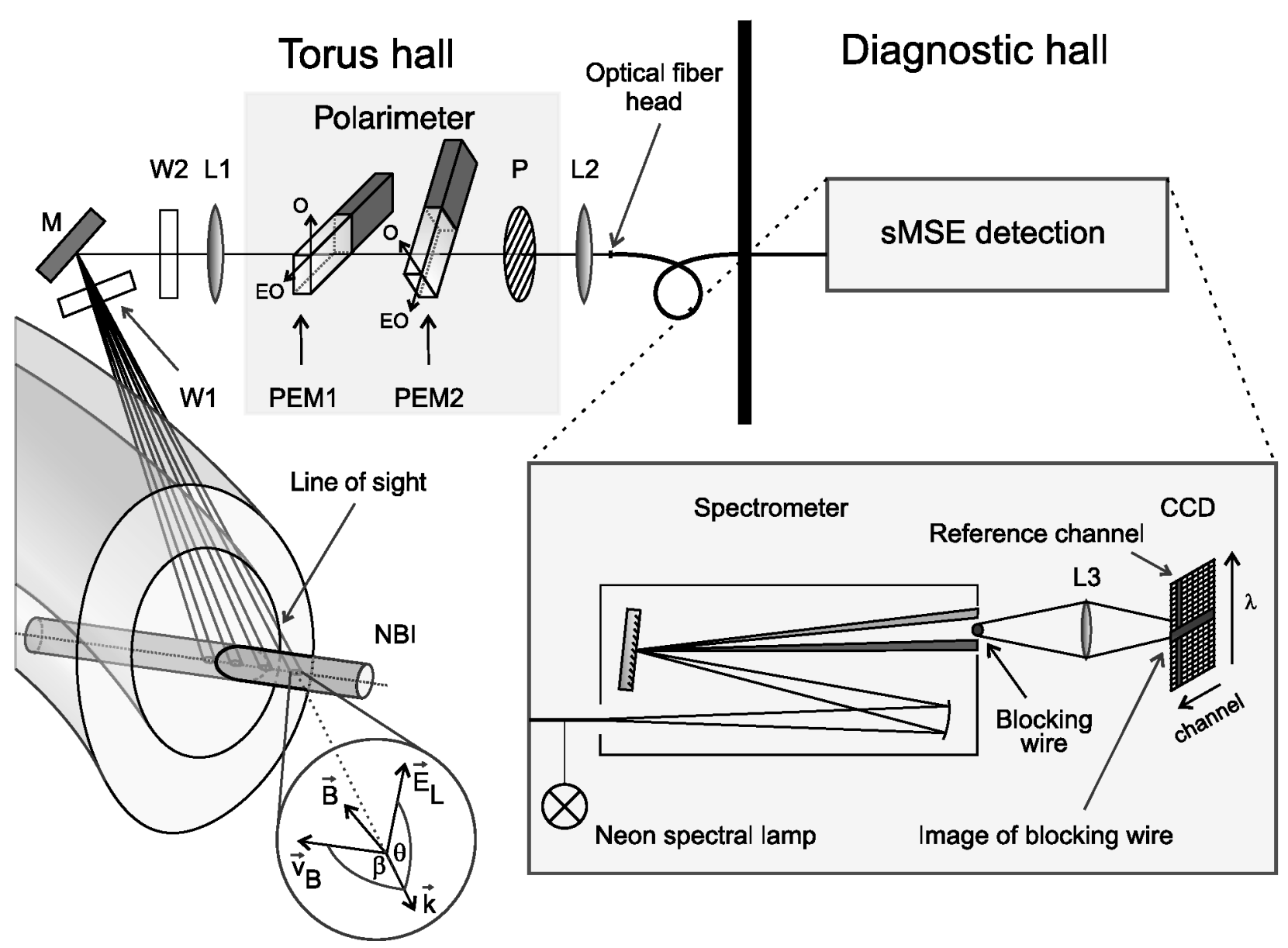

FIG. 1. Schematic figure of the sMSE set-up at ASDEX Upgrade. NBI = Neutral beam injector, $\mathrm{M}=\mathrm{mirror}, \mathrm{W} 1$ and $\mathrm{W} 2=$ cover and vacuum windows, L1, L2 and L3 = lens systems, PEM1 and PEM2 = photo-elastic modulators, P = polariser, CCD = charge-coupled device, $\overrightarrow{\mathrm{B}}=$ magnetic field, $\overrightarrow{\mathrm{v}}_{\mathrm{B}}=$ beam velocity, $\overrightarrow{\mathrm{E}}_{\mathrm{L}}=$ Lorentz field, $\overrightarrow{\mathrm{k}}=$ direction of line of sight

emission, detected by the CCD-chip, is plotted channel and spectrally resolved.

The forward modelled data have been fitted to the experimental data, by optimizing the model parameters. The fit is based on the minimization of $\chi^{2}$ where, $\chi^{2}=\sum_{i}\left(D_{i}-d_{i}\right)^{2} / \sigma_{i}^{2}$ applying the Nelder-Mead simplex algorithm. $\left(D_{i}-d_{i}\right)$ denotes the residual $\epsilon$ of the fit at the $i$ th pixel. The pixel and channel dependent error $\sigma_{i}$ of the data has been determined by calibration measurements at varying radii. The goodness-of-fit per pixel, $\chi$, in the lower subplot of fig. 2 shows that the forward model gives a very accurate description of the experimental data leading to normalized values of $\chi_{N}^{2} \approx 2.3$ (reduced by the number of points to be fitted: $\left.\chi_{N}^{2}=1 / N \sum_{i}\left(D_{i}-d_{i}\right)^{2} / \sigma_{i}^{2}\right)$.

\section{A. Forward model of MSE spectrum}

1. Basic model $\left(\mathbf{d}^{(1)}\right)$ :

Following the experimental observation in 2 the data on the detector $(D)$ can be described by eq. 6 and is composed of the following contributions: the Stark spectrum $\left(d_{\mathrm{MSE}}\right)^{13,14}$, the active CX line $\left(d_{\mathrm{CX}}\right)$, a continuous background, e.g. Bremsstrahlung radiation, $\left(d_{\mathrm{Bg}}\right)$, and impurity lines $\left(d_{\text {Imp }}\right)$. The emission from the cold plasma edge is suppressed by the aforementioned blocking wire and does not need to be considered here:

$$
d^{(1)}=d_{\mathrm{MSE}}+d_{\mathrm{CX}}+d_{\mathrm{Bg}}+d_{\mathrm{Imp}}
$$

The model of the Stark spectrum considers all 15 ( $\sigma$ and $\pi$ ) Stark components. The spectral profile function is constructed by a Gaussian. Due to the occurrence of beam particles of three different energies, three Stark spectra are modelled using the amplitude, $A_{\mathrm{b}_{i}}$, line posi- 


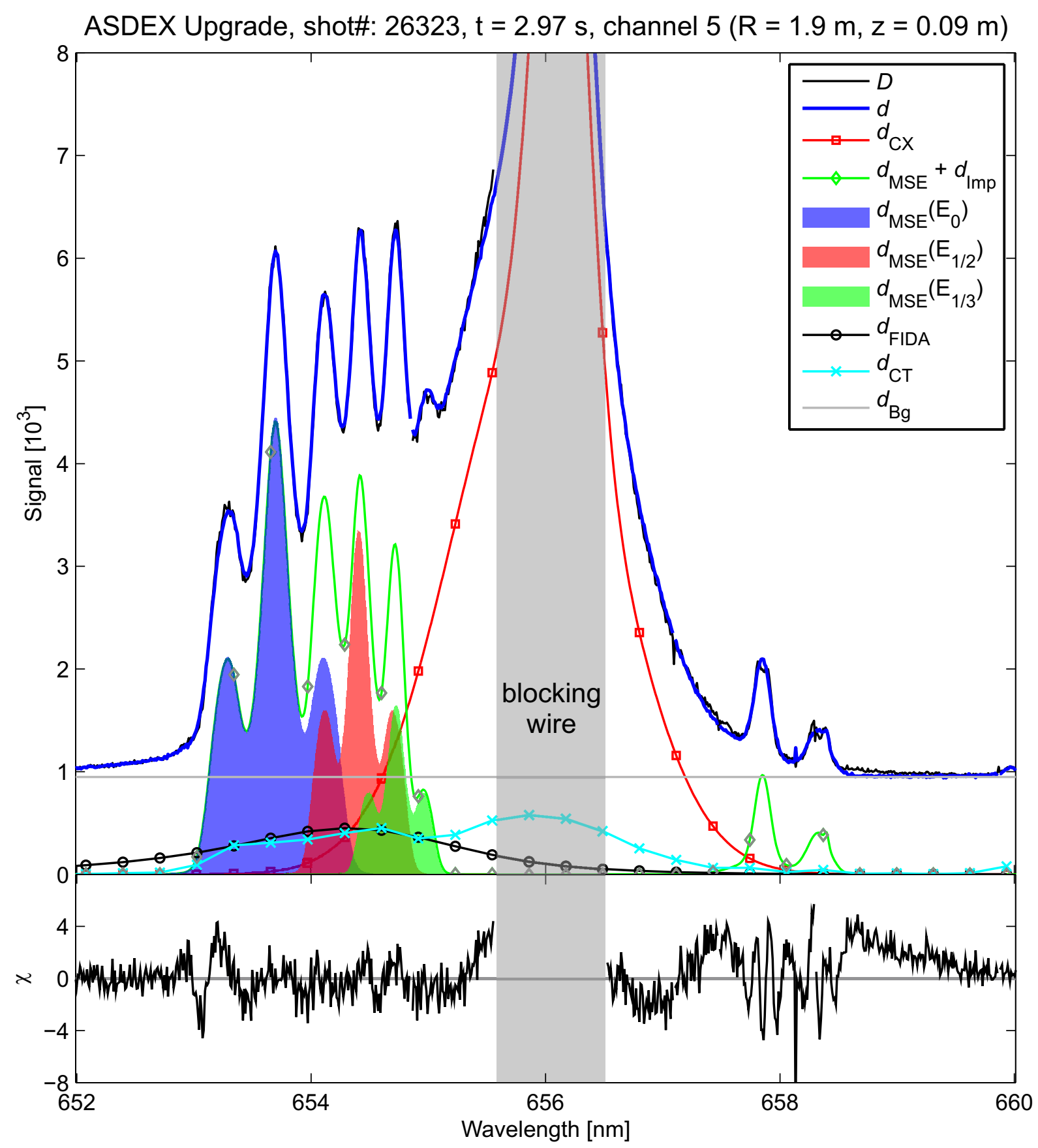

FIG. 2. Top plot: Experimental data from the ASDEX Upgrade beam emission spectrum $D$ (bold line), modelled spectrum $d$ (dashed line), consisting of $D_{\mathrm{CX}}$ component (square), $D_{\mathrm{MSE}}+D_{\mathrm{Imp}}$ line (diamond), $D_{\mathrm{FIDA}}$ (circle), $D_{\mathrm{CT}}(\mathrm{x}), D_{\mathrm{Bg}}$ line (grey). The filled area represent the calculated MSE spectra for the full (blue), half (red) and third (green) energy component. Bottom plot: $\chi$ as a measure for the goodness-of-fit.

tion, $\lambda_{\mathrm{MSE}_{i, \pi, \sigma}}$, and the quantity of interest $T_{p}^{m}=T_{\pi} / T_{\sigma}$ :

$$
\begin{aligned}
d_{\mathrm{MSE}}= & \sum_{i=1}^{3} A_{\mathrm{b}_{i}}\left(T_{\sigma} \sum_{\pi} A_{\pi} \exp \left[-\frac{1}{2}\left(\frac{\lambda-\lambda_{\mathrm{MSE}_{i, \pi}}}{\sigma_{\mathrm{MSE}}}\right)^{2}\right]\right. \\
& \left.+T_{\pi} \sum_{\sigma} A_{\sigma} \exp \left[-\frac{1}{2}\left(\frac{\lambda-\lambda_{\mathrm{MSE}_{i, \sigma}}}{\sigma_{\mathrm{MSE}}}\right)^{2}\right]\right)
\end{aligned}
$$

The Einstein coefficients $A_{\pi, \sigma}$ for the $\pi$ and $\sigma$ lines of the Stark spectrum are taken from ${ }^{10}$. The width $\sigma_{M S E}$ is mainly affected by the beam width (Doppler effect) and the instrument function. The correction of non-statistical distribution of the Stark sub-levels is made in a later step by introducing the correction factor, $c_{n s}$, as explained in sec. II. 


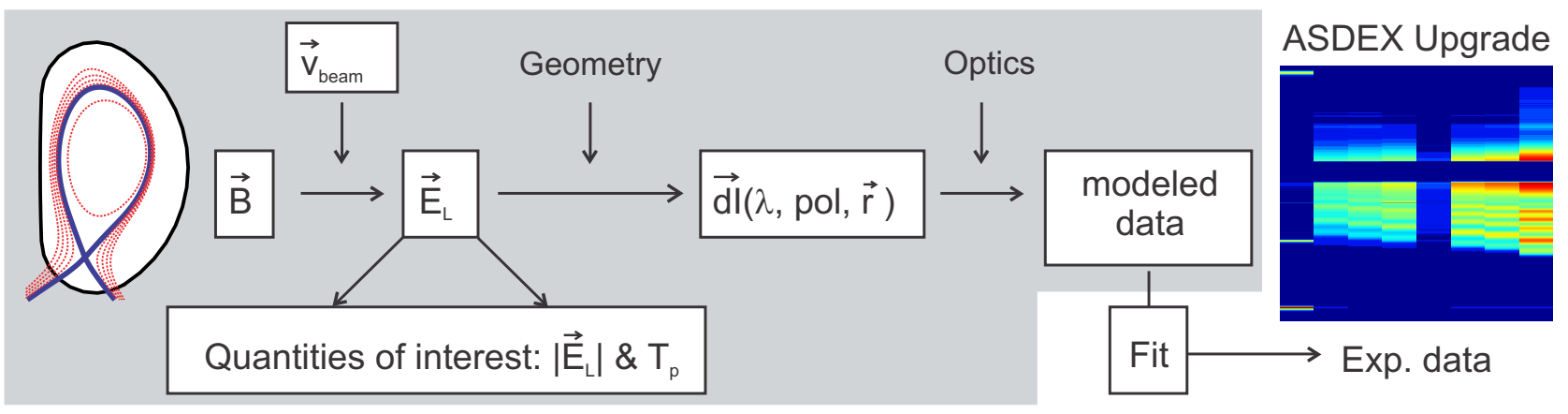

FIG. 3. Schematic flow-chart representing the forward model of sMSE measurements and the fit to experimental data. $\overrightarrow{\mathrm{B}}=$ magnetic field, $\overrightarrow{\mathrm{v}}_{\text {beam }}=$ beam velocity, $\overrightarrow{\mathrm{E}}_{\mathrm{L}}=$ Lorentz field, $T_{p}=$ Stark line ratio, $\overrightarrow{\mathrm{dI}}=$ local emission coefficient - dependent on wavelength $(\lambda)$, polarization state $(\mathrm{pol})$ and spatial position $(\overrightarrow{\mathrm{r}})$. The modelled data are fitted to experimental data. The modelled data describing the beam emission are fitted to experimental data detected by the CCD-chip (color coded plot).

The background and CX components (pedestal and active CX emission) were found to be well described by a constant $\left(A_{\mathrm{Bg}}\right)$ and two overlapping Gaussians as functions of the wavelength $\lambda$ :

$$
\begin{aligned}
d_{\mathrm{CX}}(\lambda)= & \sum_{i=1}^{2} A_{\mathrm{CX}_{i}} \sqrt{\frac{m_{\mathrm{CX}_{i}} c^{2}}{2 \pi k_{B} T_{\mathrm{CX}} \lambda_{\mathrm{CX}_{i}}^{2}}} \\
& \times \exp \left[-\frac{1}{2} \frac{m_{\mathrm{CX}_{i}} c^{2}}{k_{B} T_{\mathrm{CX}_{i}}}\left(\frac{\lambda-\lambda_{\mathrm{CX}_{i}}}{\lambda_{\mathrm{CX}_{i}}}\right)^{2}\right] \\
d_{\mathrm{Bg}}=A_{\mathrm{Bg}} &
\end{aligned}
$$

with $k_{B}$ being the Boltzmann constant and $c$ the speed of light. The widths of the Gaussians can be assigned depending on temperature and rotation velocity, which also effects the shift, reflected by $\lambda_{C X}$. At the central channel a temperature of about $T_{C X} \approx 3.5 \mathrm{keV}$ was determined for the active CX emission line, which is in the range of values determined by kinetic measurements, cf. fig. 7 . The line is shifted to $\lambda_{C X} \approx 655.83 \mathrm{~nm}$. The pedestal line has a temperature of about $T_{C X}=0.3 \mathrm{keV}$ and is almost unshifted. $A_{\mathrm{CX}_{i}}$ denotes the respective amplitudes as a measure for the number of particles. $m_{\mathrm{CX}}$ represents the atomic mass of the CX particle (deuteron).

Although not contributing to the MSE spectrum but for completion of the basic model for the observed spectrum an expression of both impurity carbon lines is given for completion. Here, again sufficAt tient accuracy is achieved when modelling these in a similar fashion to the $\mathrm{D}_{\alpha}$-CX lines, using the temperature, mass, line position and amplitude $\left(T_{\operatorname{Imp}_{i}}, m_{\operatorname{Imp}_{i}}, \lambda_{\operatorname{Imp}_{i}}, A_{\operatorname{Imp}_{i}}\right)$ :

$$
\begin{aligned}
d_{\operatorname{Imp}}= & \sum_{i=1}^{4} A_{\operatorname{Imp}_{i}} \sqrt{\frac{m_{I_{m p}} c^{2}}{2 \pi k_{B} T_{\operatorname{Imp}_{i}} \lambda_{\operatorname{Imp} p_{i}}^{2}}} \\
& \times \exp \left[-\frac{1}{2} \frac{m_{\operatorname{Imp}_{i}} c^{2}}{k_{B} T_{\operatorname{Imp}_{i}}}\left(\frac{\lambda-\lambda_{\operatorname{Imp}_{i}}}{\lambda_{\operatorname{Imp}_{i}}}\right)^{2}\right]
\end{aligned}
$$

The results of the fit with the basic forward model $\left(d^{(1)}\right)$ have indicated residuals which are due to experimental aspects described Sec. IV A 2 b.

\section{Extensions $\left(d^{(2)} \ldots d^{(7)}\right)$}

\section{a. Hardware based extensions of the forward model}

The data $(d)$ on the CCD-chip are given in pixels and must be mapped onto a wavelength scale. For this purpose natural lines from a Neon spectral lamp are detected on one channel. For the proof-of-principle a linear pixel to wavelength mapping using two Neon lines $\left(\lambda_{N e 1}=650.65 \mathrm{~nm}, \lambda_{N e 2}=659.87 \mathrm{~nm}\right)$ was sufficient ${ }^{17}$. To improve the accuracy of the wavelength mapping a quadratic dispersion relation was applied by using three natural Neon lines $\left(\lambda_{N e 1}=650.65 \mathrm{~nm}, \lambda_{N e 2}=\right.$ $\left.653.26 \mathrm{~nm}, \lambda_{N e 3}=659.87 \mathrm{~nm}\right)$. Additionally, a channel dependent shift of the wavelength scale $\left(\Delta \lambda_{0}\right)$ due to imperfections of the optics was added ${ }^{18}$. It turns out that the grating of the spectrometer is sensitive to small changes in the ambient temperature. To allow for the resulting variation in dispersion, the dispersion relation is calculated with each plasma discharge. Thus the modelled data are calculated by: $d^{(2)}=d^{(1)}\left(\lambda(\right.$ pixel $), \Delta \lambda_{0}($ channel $\left.)\right)$.

Since a frame transfer CCD-camera is used, smearing on the detector is generated during each frame transfer (vertical shift). This adds onto all spectra on the CCD-chip and needs to be accounted for in the model: $d^{(3)}=d^{(2)}+d_{\mathrm{CT}}$. The smearing between the channels on the CCD-chip is estimated by combining the calibration data obtained from a covered channel, $d_{C T_{0}}$, with a channel dependent binning factor, $C_{b i n}$, (to gain higher signals several rows are binned to one channel) and the model parameter for smearing, $C_{s m}$ : $d_{C T}=d_{C T_{0}} \cdot C_{b i n} \cdot C_{s m}$.

\section{b. Experimentally based extensions of the forward} model

Additionally aspects taken into account are non-ideal beam grids which results in focus astigmatism of the 
beam and the FIDA signal.

The first aspect is deviations of beam direction and width between the three energy components in the applied MSE geometry. This can also be observed in beam-into-gas calibration experiments ${ }^{19}$. Here, no magnetic field is applied during neutral beam injection into a gas. The observed spectrum consists of three Doppler shifted beam emission lines. Each of these lines belongs to a beam energy component and does not overlap with the others, since they are purely Gaussian shaped due to the absence of magnetic field. Thus, separate widths $\left(\sigma_{M S E}\right.$ in eq. 7 becomes $\sigma_{M S E_{i}}$, with $\left.i=\{1,2,3\}\right)$ and deviations in positions $\left(d E_{i}\right.$, with $\left.i=\{1,2,3\}\right)$ can be calculated and incorporated into the forward model for each beam energy component: $d^{(4)}=d^{(3)}\left(\sigma_{i}\right)$ and $d^{(5)}=d^{(4)}\left(d E_{i}\right)$, respectively.

The broad FIDA signal overlaps with the whole MSE spectrum but is of low intensity ${ }^{20}$. In order to avoid the high modelling effort required for the small contribution of the FIDA signal, this component is approximated by two overlapping Gaussians of low heights at distinctly different wavelengths and with a large width of $\approx 1.5 \mathrm{~nm}$ (channel dependent): $d^{(6)}=d^{(5)}+d_{\text {FIDA }}$.

$$
d_{\mathrm{FIDA}}=\sum_{i=1}^{2} A_{\mathrm{FIDA}_{i}} \exp \left[-\frac{1}{2}\left(\frac{\lambda-\lambda_{\mathrm{FIDA}_{i}}}{\sigma_{\mathrm{FIDA}_{i}}}\right)^{2}\right]
$$

c. Constraints of the forward model

The CX emission line is partly covered with only the right and left hand side wings available to be fitted, and the MSE spectrum is overlayed with the flat FIDA and smearing signals. This can cause the fit to reach a local minimum with physically unrealistic spectra. Thus, strict boundary conditions are imposed for several parameters, e.g. $T_{C X_{i}}$ and $\lambda_{C X_{i}}$ : $d^{(7)}=d^{(6)}$ (boundary cond.)

\section{B. Model and fit validation}

To assess the enhancements of the different parts of the extended forward model (hardware and experimentally based parts as well as constraints) and to compare these approaches with the mathematical one (multi-Gaussian based simplex model) the reduced goodness-of-fit was calculated for each part. In these calculations the influence of the increase of the number of free parameters, which are outlined in the right table of fig. 4 is included. In the left plot of fig. 4 the calculated $\chi_{N}^{2}$ for each model are presented for three different channels. It can be seen that even the basic physically motivated approach leads to much higher accuracy than the multi-Gaussian model. The use of a more accurate quadratic dispersion relation instead of the linear one and consideration of the smearing between the channels in the forward model reduces $\chi_{N}^{2}$ significantly. Further reductions of $\chi_{N}^{2}$ are achieved when taking into account experiment based aspects as deviations of beam width and beam energy for each beam energy component and the FIDA signal. The fit of the modelled data with constraints, especially for fit parameters describing the CX components prevent the fit from running into local minima and thus leads to better fit results with higher accuracy.

The reason for the improvements by fitting to a forward model is the matching of the spectral features of interest to the physical model making use of the entire spectrum. Fitting of individual lines (even with constraints) is much more sensitive to experimental errors and (local) background subtraction.

As outlined previously, the MSE spectrum is described by a model with only two quantities $\left(E_{L}, T_{p}\right)$ and a set of fit parameters. However, the forward model uses all fit parameters leading to a complicated multivariate likelihood function underlying the fit procedure.

For an error discussion we assess a low dimensional projection of the likelihood function, cf. fig. 5 and 6 . The projections are chosen to assess both the uncertainties of the fit parameter and their respective correlations. For visualisation, both the log-likelihood and the chosen model parameters are normalized with respect to the best fit value. In this representation the best-fit value is for all plots at $(x=1, y=1)$ with a minimum $\chi_{N}^{2}$ of 1.95 .

The error bars in fig. 5 and 6 show the $2 \sigma$ error of the fit parameters. The ellipses in the two-dimensional projection show the $2.3 \cdot \sigma$ (containing $68.3 \%$ of the data) and $11.8 \cdot \sigma$ (containing $99.73 \%$ of the data) iso-contours, respectively. The tilt of the contours reflects the linear correlation of the model parameters displayed. The chosen parameters are the measurand quantities $E_{L}$ and $T_{p}$ and some model parameter relevant to experimental set-up $\left(A_{b}, \alpha\right.$ and $\left.C_{s m}\right)$. It is shown that significant correlation shown-up in the error analysis of $E_{L}$ with $\alpha$ and of $T_{p}$ with $A_{b}$ and $C_{s m}$.

The Lorentz field was found to be independent of $A_{b}$ and $C_{s m}$. This is indicated by the circular $\left(A_{b}\right)$ and non-inclined elliptical $\left(C_{s m}\right)$ distribution of the data. However there is a weakly negative linear correlation between $E_{L}$ and the observation angle, shown in the middle left hand plot. The negative sign follows the direction of the ellipse's main axis. To fulfil the same confidence interval, an increase in one parameter enforces a decrease in the other and vice versa. The different scaling of the $\mathrm{x}$ - and $\mathrm{y}$-axes need to be considered in the middle left plot.

$T_{p}$ is linearly and negatively correlated to the beam amplitude and smearing factor, but not connected to the observation angle. This agrees with the expectation for $T_{p}$ which depends on the intensity as well as the beam amplitude, while the observation angle depends on the wavelength. The opposite is true for the Lorentz field, which is calculated from the Stark-splitting and in consequence is dependent on the wavelength but not on the intensity. Hence, it is not surprising that $E_{L}$ and $T_{p}$ are not correlated to each other, as reflected in fig. 6 . Finally, the estimated precision of $E_{L}$ is $\pm 0.02 \%$ at the 
(a)

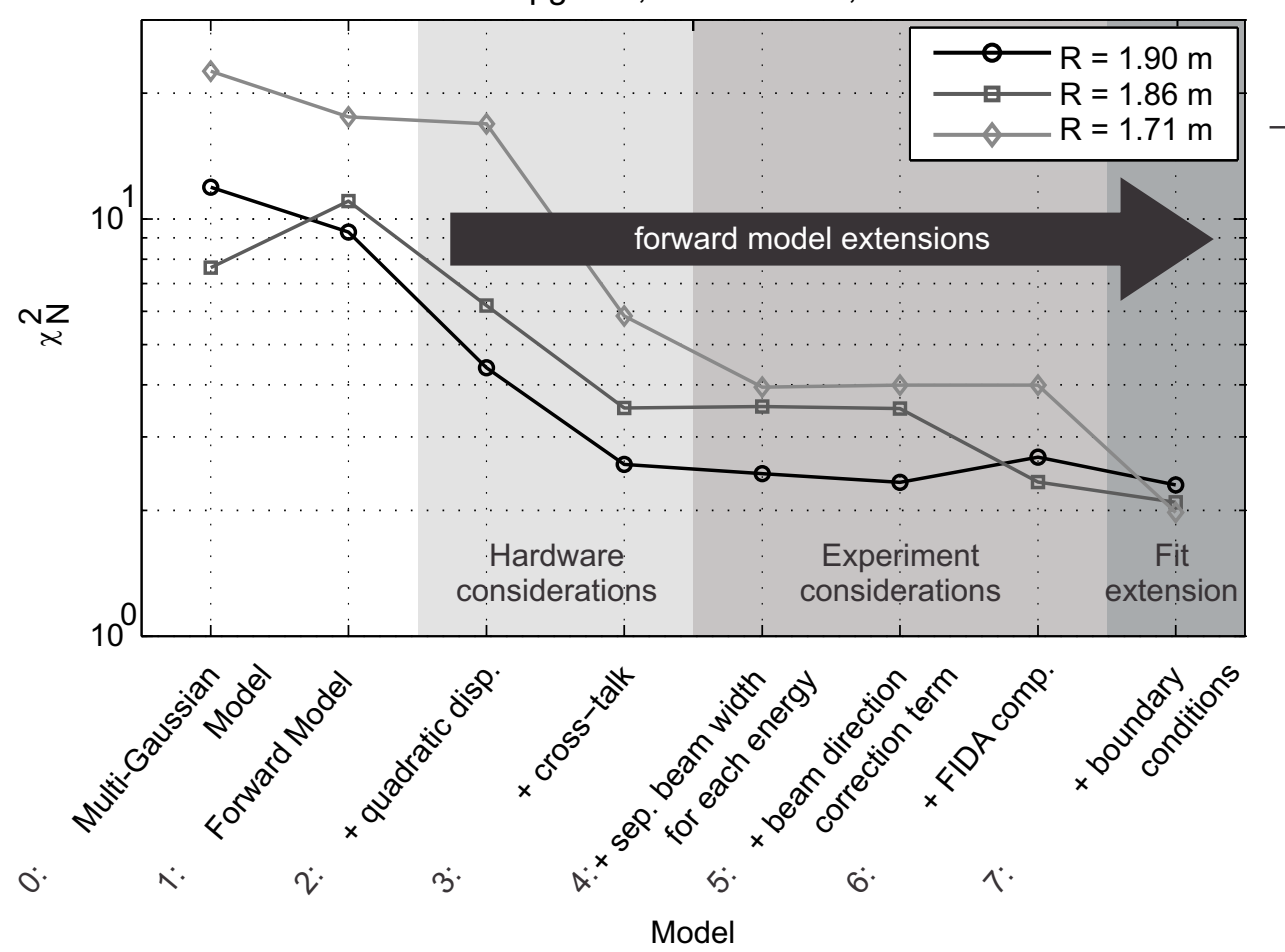

(b)

\begin{tabular}{c|c} 
Model & $\begin{array}{c}\text { Number of free } \\
\text { Parameters } f_{i}\end{array}$ \\
\hline $0:$ & 17 \\
1: & 35 \\
2: & 39 \\
3: & 40 \\
4: & 43 \\
5: & 45 \\
6: & 51 \\
7: & 51
\end{tabular}

FIG. 4. (a): Comparison between the multi-Gaussian based simplex model, the forward model and the extended forward model, latter regarded for its components separately $\left(d^{(1)}, d^{(2)}=d^{(1)}+\right.$ quadratic disp., $d^{(3)}=d^{(2)}+d_{C T}, d^{(4)}=d^{(3)}+$ separated width for each beam energy component, $d^{(5)}=d^{(4)}+$ deviation term for variation in beam direction, $d^{(6)}=d^{(5)}+d_{\text {FIDA }}$, $d^{(7)}=d^{(6)}+$ boundary conditions) for three different channels. (b): At the right table the number of free parameters, $f_{i}$, considered in $\chi_{N}^{2}\left(N=\right.$ \#datapoints $\left.-f_{i}\right)$ are outlined for each model.

outermost channel and rises to $\pm 0.09 \%$ at the innermost channel with respect to the plasma core. The increase in uncertainty can be explained by the decrease in the signal-to-noise ratio due to beam-stopping.

\section{MEASUREMENT OF THE MAGNETIC FIELD}

As an example of the application of the MSE diagnostic fig. 7 displays results from sMSE measurements for the ASDEX Upgrade discharge \#26323 $\left(B_{\text {tor }}=2.48 \mathrm{~T}\right.$, $I_{p}=0.8 \mathrm{MA}$ ). The timings of the heating power by neutral beam injection (NBI) and electron heating (ECRH) are displayed in the upper panel of fig. 7 showing a stepwise increase in heating power up to $10.8 \mathrm{MW}$. The heating is provided by four 2.5 MW NBI sources: the more tangentially off-axis deposited heating power of NBI6, the more radially on-axis heating power of NBI8 and NBI5 are added to beam heating of NBI3 used for th sMSE diagnostic. To prevent Wolfram density peaking in the plasma center ECRH heating power of $0.8 \mathrm{MW}$ is applied. The discharge is fuelled by gas puffing and the electron density is $n_{0 e}=6.3 \ldots 6.7 \times 10^{19} \mathrm{~m}^{3}$. The second row in fig. 7 shows the central electron temperature and density, estimated by integrated data analysis
$\left(\mathrm{IDA}^{21}\right)$, along with ion temperature measurements from charge exchange recombination spectroscopy ${ }^{22}$.

The third row in fig. 7 reflects stepwise increasing heating power in the apparent intensity ratio, $T_{p}^{n s}$. This effect increases towards the plasma core and innermost observation channel (at $R=1.74 \mathrm{~m}$ and $z=0.09 \mathrm{~m}$ ). The Lorentz field also changes when the maximum heating power is applied as seen in the fourth panel of fig. 7. This effect is less obvious than the change in $T_{p}^{n s}$ but is still visible. In order to examine the temporal evolution of $T_{p}^{n s}$ and $E_{L}$, the bottom row of fig. 7 shows more details along with exponential fits to the temporal evolution. The Lorentz field increases on time scales of $\delta_{t}\left(E_{L}\right) \approx 80 \mathrm{~ms}$ when switching off NBI5 (T2), whereas the polarization fraction relaxes with $\delta_{t}\left(T_{p}^{n s}\right) \approx 120 \mathrm{~ms}$. This figure shows typical NBI slowing down times of about $150 \mathrm{~ms}$. These findings indicate the temporal resolution of the sMSE for equilibrium reconstruction.

In the phase of greatest heating power, an increase in the central pressure can be observed from kinetic measurements, as well as a decrease after switching off NBI5 (second row of fig. 7). The kinetic pressure change (taken from kinetic measurements) is compared with the (dia)magnetic pressure change derived from the sMSE measurement $\left(\Delta p \approx-\Delta E_{L} / E_{L} \cdot B^{2} / \mu_{0}\right)$ for two time points in fig. 8. These time points indicate 


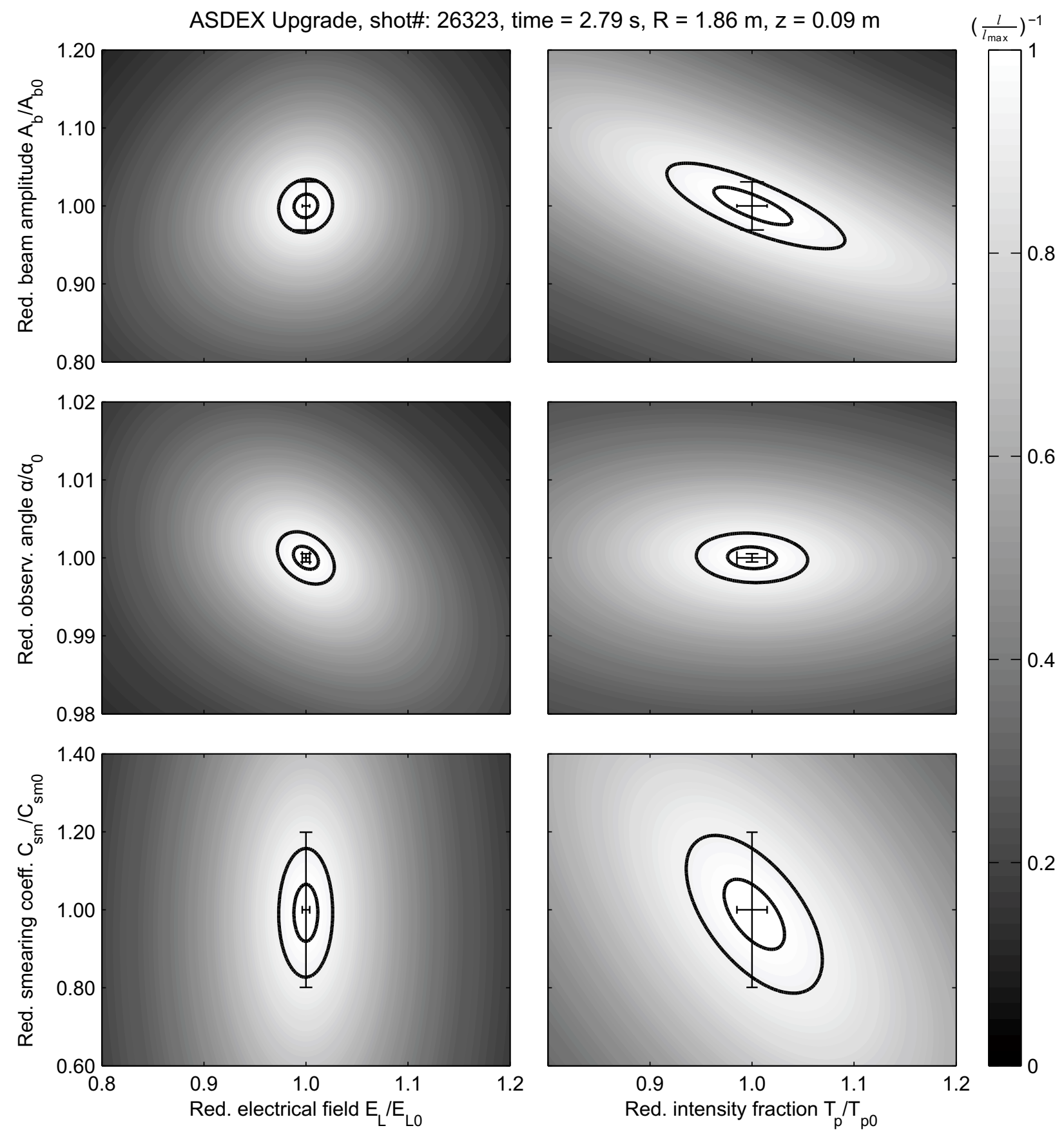

FIG. 5. Results of the error analysis of the MSE spectrum (grey-color coded is the log-likelihood $l$ - normed with respect to $\left.\operatorname{maximum}\left(l / l_{\max }\right)^{-1}\right)$

different heating scenario transitions which are marked in fig. 7 with $\mathrm{T}_{O N}$ : before and after switching NBI5 on $(t=2.91 \mathrm{~s} \rightarrow t=3.42 \mathrm{~s})$ and $\mathrm{T}_{O F F}:$ before and after switching NBI5 off $(t=3.42 \mathrm{~s} \rightarrow t=4.14 \mathrm{~s})$. The radial dependency shows good agreement of these two independent methods for estimating pressure change. Both techniques show small pressure variations in the outermost channel and an increase in the pressure change towards the plasma core. The pressure changes for both heating scenaris transistions are about the same for all channels $\left(\Delta p\left(T_{O N}\right) \approx-\Delta p\left(T_{O F F}\right)\right)$.

The error bars indicate the $1 \sigma$ interval of the error considering uncertainties in $B_{t o r}$ and $E_{L}$. The latter is estimated by averaging over a period of almost constant plasma conditions (about $30 \mathrm{~ms}$ after the end of the slowing down phase) during and after high heating power, $t=3.18 \ldots 3.60 \mathrm{~s}$ ( $\widehat{=}$ high power) and $t=3.78 \ldots t=4.20 \mathrm{~s}$ (金low power).

Possible reasons for the magnetic pressure variation can be extracted from the radial profiles of $E_{L}$ for the two 


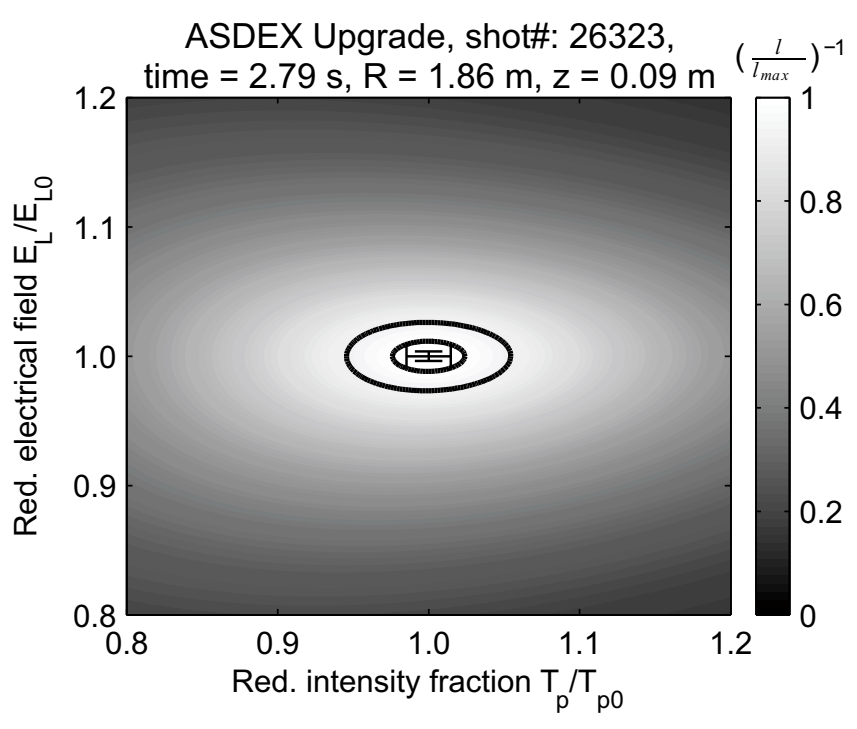

FIG. 6. Correlation analysis $T_{p}$ and $E_{L}$ (grey-color coded is the $\log$-likelihood $l$ - normed with respect to maximum $\left.\left(l / l_{\max }\right)^{-1}\right)$.

discharge periods (fig. 9) in conjunction with results of equilibrium calculations. The equilibrium was calculated solving the Grad-Shafranov equation with CLISTE constrained by inductive magnetic measurements and a pressure profile which has been calculated with data from the kinetic measurement and from IDA. The fraction of the fast ion pressure is neglected. Moreover, the q-profile was calculated with the determination of the $q=1$ surface of the sawtooth inversion radius.

There is almost no change in the Lorentz field in the outermost channel (at $R=1.99 \mathrm{~m}$ ), cf. fig. 9. In contrast, the channels $R=\{1.90 \mathrm{~m}, 1.86 \mathrm{~m}, 1.77 \mathrm{~m}\}$ reflect a diamagnetic behaviour of the plasma, i. e. in the higher power phase the Lorentz field and thus $B_{\text {tor }}$ is lowered reflecting the increasing plasma diamagnetism. Even the central channel shows a small decrease in the Lorentz field. The diamagnetic behavior can be explained by the Shafranov shift shown by the vertical lines in fig. 9 and fig. 11 in the high power phase. The magnetic axis shifts with decreasing NBI power from about $R \approx 1.729 \mathrm{~m}$ to about $R \approx 1.715 \mathrm{~m}$ towards the high field side. The resulting shift of the pressure profile leads to the variation in the Lorentz field which is calculated with an uncertainty of $\pm 0.12 \%$.

In fig. 10 absolute values of the measured Lorentz fields from the Stark-splitting are compared with values derived from magnetic field reconstruction of the CLISTE code and the neutral beam geometry ${ }^{23}$.

The difference in the outcome of both methods is less than $1.5 \%$ in absolute values. In addition, the similar shapes of the time traces of the Lorentz fields reflect a good agreement of less than $1 \%$ difference in relative values.
A comparison of $T_{p}^{n s}$ with the pitch angle in MSE geometry, $\gamma_{C l}$, in fig. 11 shows $T_{p}^{n s}$ to be an experimental indication for the direction of $E_{L}$ and $B$ in the MSE geometry. The profiles of $T_{p}^{n s}$ (upper subplot) and $\gamma_{C l}$ (middle subplot) are increasing with increasing beam power (fig. 11). This effect gets stronger towards the core and indicates a change in the poloidal field.

In fig. 12 the variation of the quantisation axis $\left(E_{L}\right)$ from $\vec{E}_{L 1}$ to $\vec{E}_{L 2}$ is observed with the LOS. Thus the detected $\pi_{1,2^{-}}$and $\sigma_{1,2}$ polarized emission lines and the observation angle on $\vec{E}_{L}, \theta_{1,2}$, vary for both cases. With eq. 5 follows a change in $T_{p}^{n s}$. Considering the fact that $\left|\vec{B}_{\text {pol }}\right|$ is about $10 \%$ of $\left|\vec{B}_{\text {tor }}\right|$ at the edge and much less in the plasma center it follows that $T_{p}^{n s}$ captures changes mainly in $\left|\vec{B}_{p o l}\right|$.

The magnetic variation during the two time periods can also be observed in the profiles of the safety factor $q$. These are calculated by the equilibrium solver and show a much stiffer shear of the magnetic configuration for the time after NBI was switched off, cf. lower subplot of fig. 11.

Systematic influence of polarizing optical elements on MSE polarimetry measurements are reported ${ }^{24}$. In our studies, consistently with these measurements, a discharge and spatially dependent systematic bias with respect to results from equilibrium solver, $\Delta \gamma_{\text {bias }}$, has also been found. Therefore, a characterization of the polarizing properties of the optical elements (fig. 1), such as the retardance of the polarimeter is essential. For this purpose in-vessel calibration measurements with a polarized light source were performed without plasma operation. For the specific case discussed here $((t=2.92 \mathrm{~s}, R=1.91 \mathrm{~m}$ and $z=0.09 \mathrm{~m})$ the bias was decreased from $\Delta \gamma_{b i a s}=17.17^{\circ}$ to $\Delta \gamma_{c a l}=-2.78^{\circ}$ with a precision of $\delta \gamma_{0}= \pm 3.68^{\circ}$.

A second systematic effect is the non-statistical distribution of the Stark sub-levels. Including this effect decreases the bias to $\Delta \gamma_{\text {non-stat }}=0.18^{\circ}$ and $\delta \gamma_{\text {non-stat }}= \pm 3.65^{\circ}$. Other cases show deviations (bias) up to $\Delta \gamma_{\text {non-stat }}=1.8^{\circ}$ with respect to CLISTE results. On the one hand, within the errorbars we find a consistent agreement between the equilibrium solver and our independent measurements. Thus, qualitative changes in the direction of the magnetic field can be detected, but the required absolute accuracy for the current $j$-profile reconstruction could not be achieved.

\section{CONCLUSIONS}

The implementation of a spectral Motional Stark diagnostics on ASDEX Upgrade has been reported. Fit functions based on forward modelling of the measurements have been employed to analyze the data. The best fitting forward model includes the Stark-splitting of three components of the neutral beam injection, 
ASDEX Upgrade, shot\# 26323

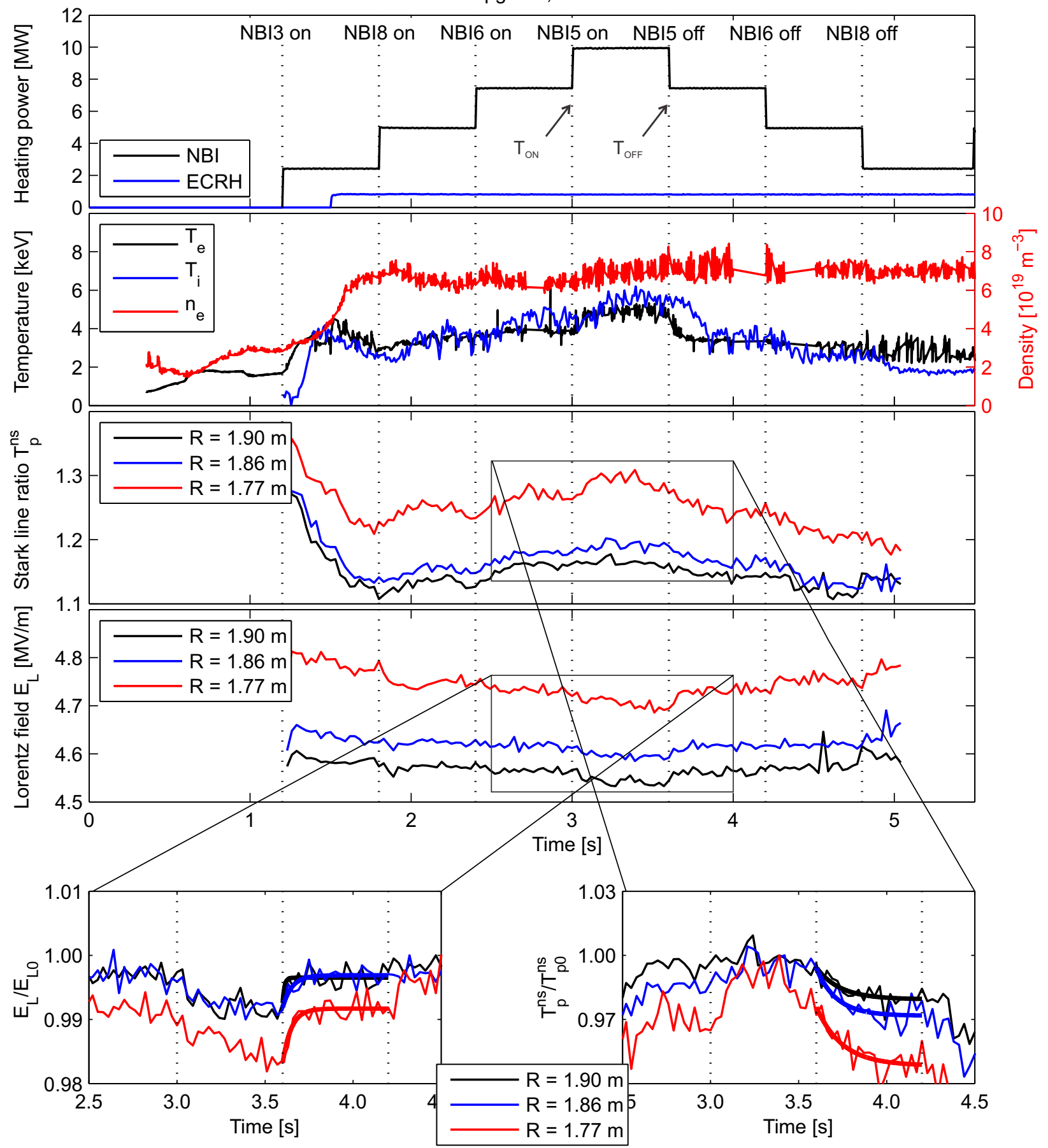

FIG. 7. sMSE results: Time traces for the measured Lorentz field $E_{L}$ and the apparent polarization ratio $T_{p}^{n s}$ (third and fourth row) for an ASDEX Upgrade discharge (sMSE observed on NBI3) and a detailed view of the quantities of interest with exponential fit for the temporal evolution (lower panel). Time traces of the port-through power of applied heating sources is shown in the first row, the second row shows the central electron temperature $\left(T_{e}\right)$ and density $\left(n_{e}\right)\left(\mathrm{IDA}^{21}\right)$ along with ion temperature measurements $\left(T_{i}\right)$ from charge exchange recombination spectroscopy. 


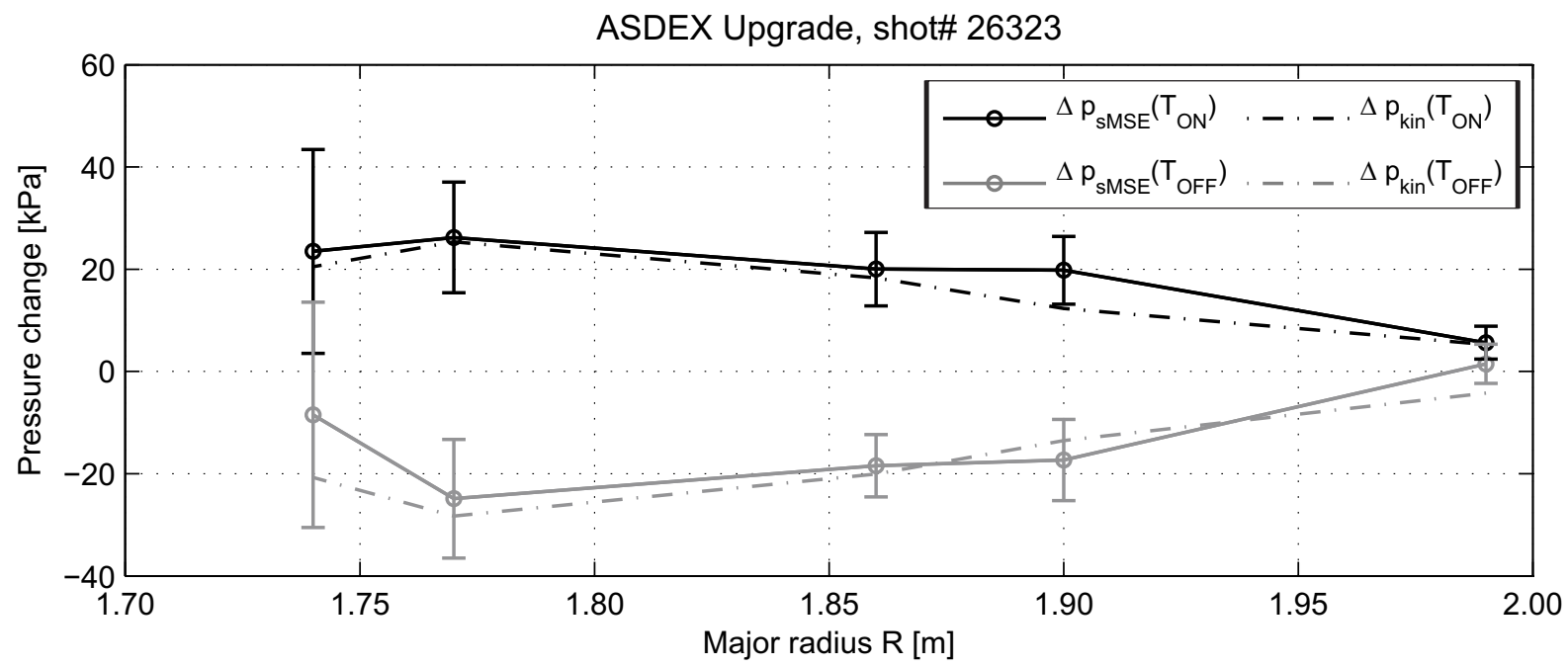

FIG. 8. Pressure change for heating scenario transition of $\mathrm{T}_{\mathrm{ON}}$ : before and after NBI5 on (black curves) and $\mathrm{T}_{\mathrm{OFF}}$ : before and after switching NBI5 off (grey curves) obtained from the fitted Lorentz field (bold lines with circle-like symbol) and from kinetic measurements (dashed lines)for different radial positions.

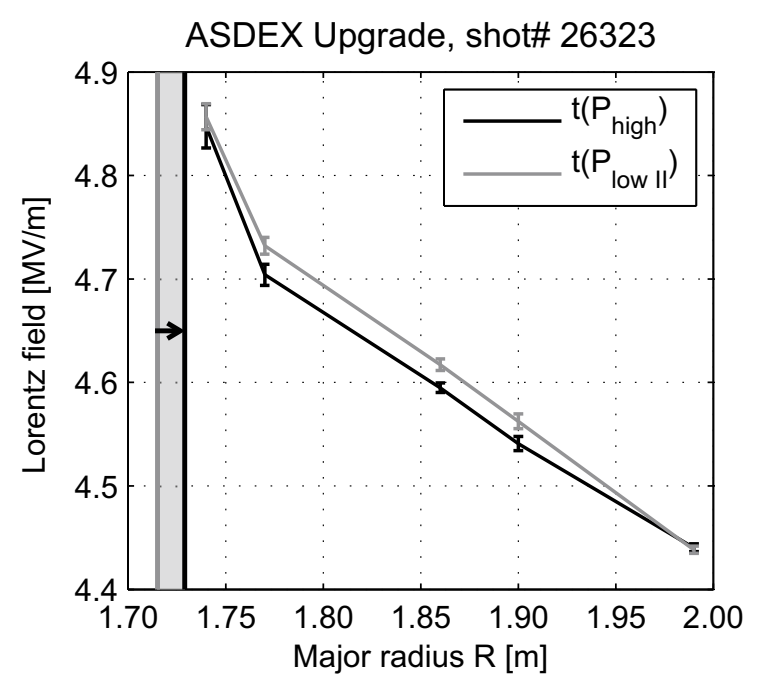

FIG. 9. Radial profile of fitted Lorentz field during high power phase (black) and after high power phase (grey curve). The radial shift of the magnetic axis for these time points is shown by the vertical lines in the same color.

background signals including fast ion $D_{\alpha}$ emission $^{25}$ and non-statistical distributions of excited atoms ${ }^{12}$. Moreover, technical influences such as a non-linear dispersion, cross-talk of CCD pixels, deviation of beam direction and width between the three beam energy components have been shown to affect the results significantly. The forward model as introduced in this paper was as globally statistically consistent with the data as to result in a normalized chi-square of $\chi^{2} / N=1.9$. For this forward model all effects have been either physically justified or proven by additional laboratory studies. This approach is in contrast to (mathematical based) heuristic parametric models, such as a multi-Gaussian model and showed much better fitting results.

Since the sMSE employs almost the same viewing geometry as the MSE polarimetry, the findings of the forward model give indications for improvements in the polarimetry such as considerations of the influence of polarizing elements in the optical path.

The most prominent quantities of interest of the measurement, the Lorentz field and the polarization factor given by $\sigma$ - and $\pi$-polarized line intensities, have been determined quantitatively. A detailed error discussion indicates the statistical uncertainties (precision) and correlations of statistical errors in the quantities of interest and further model parameters, e.g. the beam intensity or the cross-talk in the CCD-chip. The statistical errors for the Lorentz field and the polarization factor are almost independent. The determination of $E_{L}$ is not significantly correlated with the intensity ratio. This independence of polarization states is an advantage compared to MSE polarimetry especially in devices with high densities and temperatures, such as ITER. In ITER plasmas plasma facing mirrors are expected to be coated which can change the polarization state of the emitted light significantly ${ }^{26-28}$. Figures for the accuracy have been determined by systematic analyses of a well diagnosed plasma discharge at different heating scenarios, from comparisons with equilibrium modelling $\left(\right.$ CLISTE $\left.^{2}\right)$ and by comparison with independent measurements of the plasma pressure.

The Lorentz field reflecting the total magnetic field 


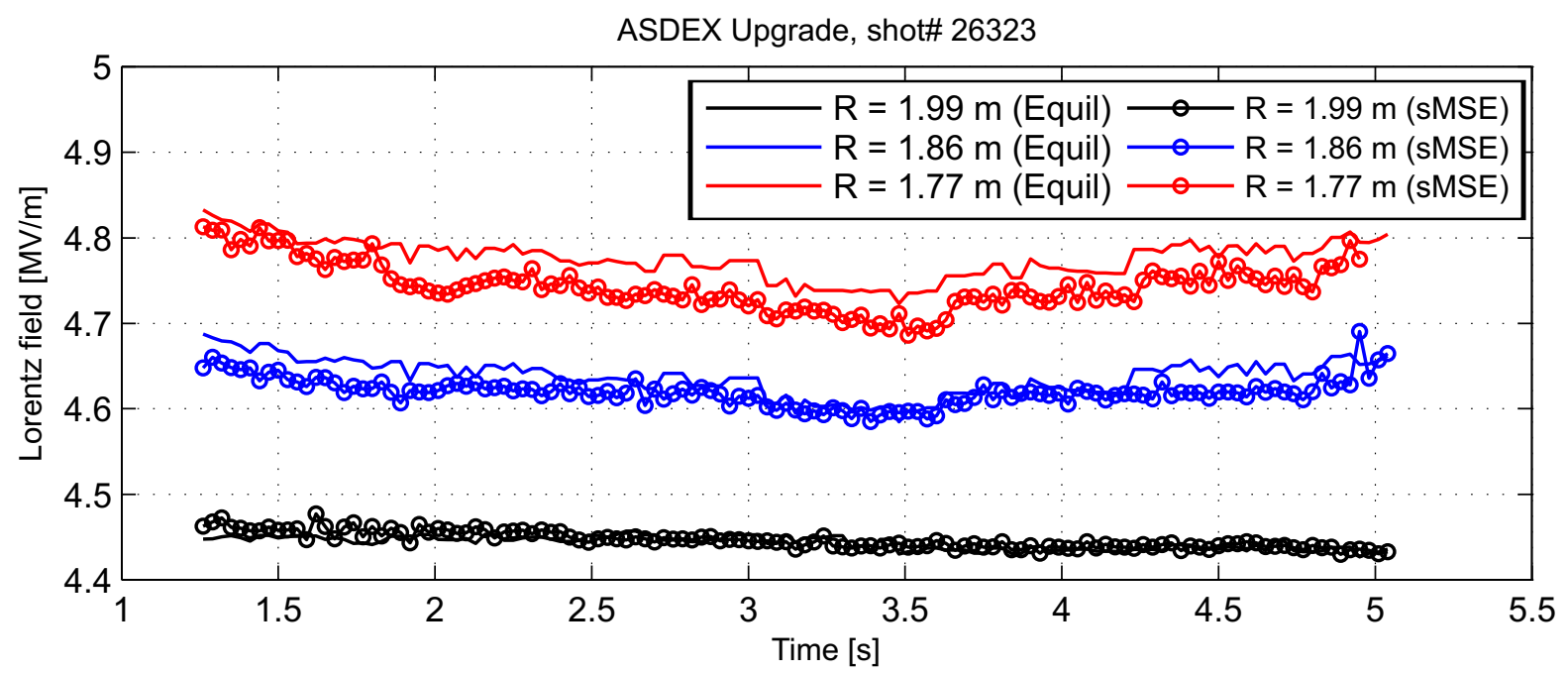

FIG. 10. Comparison of a set of time traces of the fitting parameter $E_{L}$ (bold line with circle-like symbol) with the outcome of the CLISTE equilibrium code for three different radial positions (bold line).

has been determined reliably to a channel dependent accuracy of $<1.5 \%$ and a channel dependent precision of $<0.1 \%$. These figures allow determination of the diamagnetic effect. The polarisation factor indicates the direction of the magnetic field and can be determined with high precisions. However, the accuracy suffers from systematic polarization effects such as from the plasma facing window. It is noted that these issues are circumvented in polarimetry by additional calibration measurements which could also be used for sMSE. Nonetheless, small changes in the polarisation factor in different heating scenarios have been clearly revealed.

It is concluded that the sMSE diagnostics has proven to provide reliably magnetic field strengths and precisely changes of its direction. Indications on the impact of polarization effects suggest permanent monitoring of the polarization properties of observation optics even for polarimetry.

In order to generalize the applicability of the forward model, non-Gaussian and asymmetric line shapes of the MSE spectrum should be included. It has been shown in $^{29}$ that this effect can significantly influence the MSE spectrum.

For further physics modelling, the sMSE data will be included in equilibrium modelling in a next step.

\section{ACKNOWLEDGMENTS}

The authors would like to thank Oleksandr Marchuk for calculation of non-statistical distribution of Stark sublevels and Mike Dunne for equilibrium modelling. Furthermore, the authors are grateful to the members of the Wendelstein 7-X (W7-X) Team for valuable discussions.
${ }^{1}$ W.W. Heidbrink and G.J. Sadler, Nucl. Fusion 34 535-615 (1994) ${ }^{2}$ P.J. Mc Carthy and ASDEX Upgrade Team, Plas. Phys. Control. Fusion 54 (2012) 015010

${ }^{3}$ H. Soltwisch, Rev. Sci. Instrum. 57, 1939-1944 (1986)

${ }^{4}$ F.M. Levinton, G.M. Gammel, R. Kaita, et al., Rev. Sci. Instrum. 61(10, Part2), 2914-2919 (1990)

${ }^{5}$ R.C. Wolf, L.-G. Eriksson, M. von Hellermann, et al., Nucl. Fusion 33, 1835-1847 (1993)

${ }^{6} \mathrm{P}$. Heckmann and E. Träbert, Introduction to the Spectroscopy of Atoms (North-Holland, 1989)

${ }^{7}$ B.H. Bransden and C.J. Joachain, Physics of atoms and moleculs, (Pearson Education 2003)

${ }^{8}$ D. Wroblewski, K.H. Burrel, L.L. Lao, et al., Rev. Sci. Instrum. 61, 3552-3556 (1990)

${ }^{9}$ N. Pablant, K.H. Burrell, R.J. Groebner, et al., Rev. Sci. Instrum. 81, 10D729 (2010)

${ }^{10}$ E. Schrödinger, Ann. Phys. 384, 361-376 (1926)

${ }^{11}$ W. Mandl, R.C. Wolf, M.G. von Hellermann, et al., Plasma Phys. Contrl. Fusion 35, 1373 (1993)

${ }^{12}$ O. Marchuk, Yu. Ralchenko, R.K. Janev, et al., J. Phys., 43, $011002(2010)$

${ }^{13}$ R. Reimer, A. Dinklage, J. Geiger, et al., Contrib. Plasma Phys. 50, 731-735 (2010).

${ }^{14}$ A. Dinklage, R. Reimer, R.C. Wolf, Fusion Sci. Technol. 59, 406417 (2011)

${ }^{15}$ C. Hopf and N. Rust, private communications (2011)

${ }^{16}$ E. Foley, F.M. Levinton, H.Y. Yuh et al., Nucl. Fusion 48, 085004 (2008)

${ }^{17}$ R. Reimer, A. Dinklage, R. Fischer, et al., Proceedings 38th EPS Conf. on Plasma Phys., Strasbourg (2011)P5.076

${ }^{18} \mathrm{M}$. Reich, private communication (2011)

${ }^{19}$ R. Dux, B. Geiger, R.M. McDermott, et al., Proceedings 38th EPS Conf. on Plasma Phys., Strasbourg (2011)P1.056

${ }^{20}$ Y. Luo, W.W. Heidbrink, K.H. Burrell, et al., Rev. Sci. Instrum. 41, 033505 (2007)

${ }^{21}$ R. Fischer, C.J. Fuchs, B. Kurzan, et al., Fusion Sci. Technol. 58, 675 (2010)

${ }^{22}$ R.C. Isler, Plasma Phys. Contrl. Fusion36, 171 (1994)

${ }^{23}$ W. Schneider, P. McCarthy, K. Lackner, et al., Fusion Eng. Des. 48, 127 (2000)

${ }^{24}$ T. Löbhard, Kalibrierverfahren für die Motional Stark Effekt Diagnostik am Fusionsexperiment ASDEX Upgrade, Diploma the- 


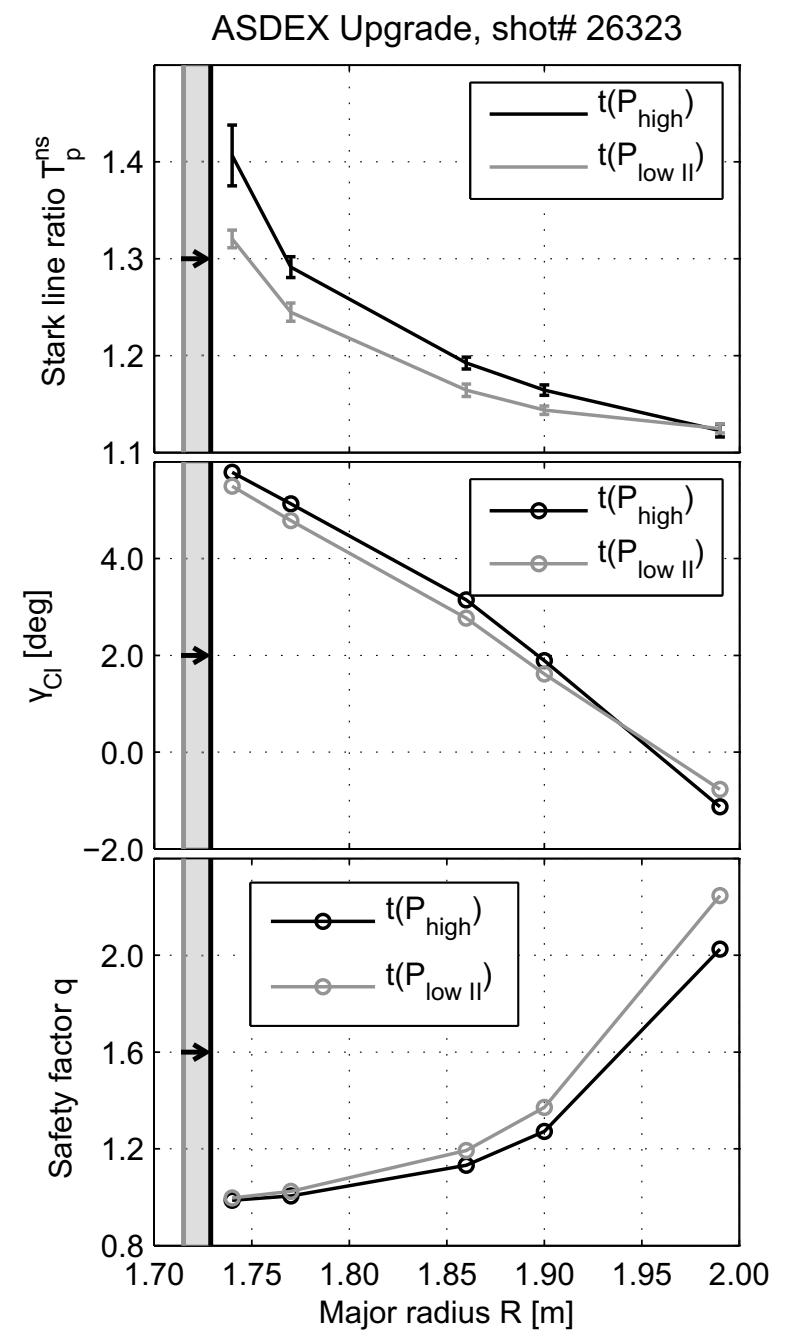

FIG. 11. Radial profile of polarization fraction (upper subplot) and equilibrium result for pitch angle in MSE geometry $\gamma_{C l}$ (middle subplot) and safety factor q (lower subplot) during high power phase (black) and after high power phase (grey curve). The radial shift of the magnetic axis for these time points is shown by the vertical lines in the same color. sis $(2011)$

${ }^{25}$ B. Geiger, M. Garcia-Munoz, W.W. Heidbrink, et al., Plasma Phys. Control. Fusion 53, 065010 (2011)

${ }^{26}$ A. Malaquias, M. von Hellermann, S. Tugiranov, et al., Rev. Sci. Instrum. 75, 3393 (2004)

${ }^{27}$ N.A. Pablant, K.H. Burrell, R.J. Groebner, et al., Rev. Sci. Instrum. 79, 10F517 (2008)

${ }^{28}$ M.A. Makowski, S.L. Allen, C.T. Holcomb, et al., Rev. Sci. Instrum. 79, 10F519 (2008)

${ }^{29}$ M.F.M. de Bock, et al., Rev. Sci. Instrum. 79, 10F524(2008) 


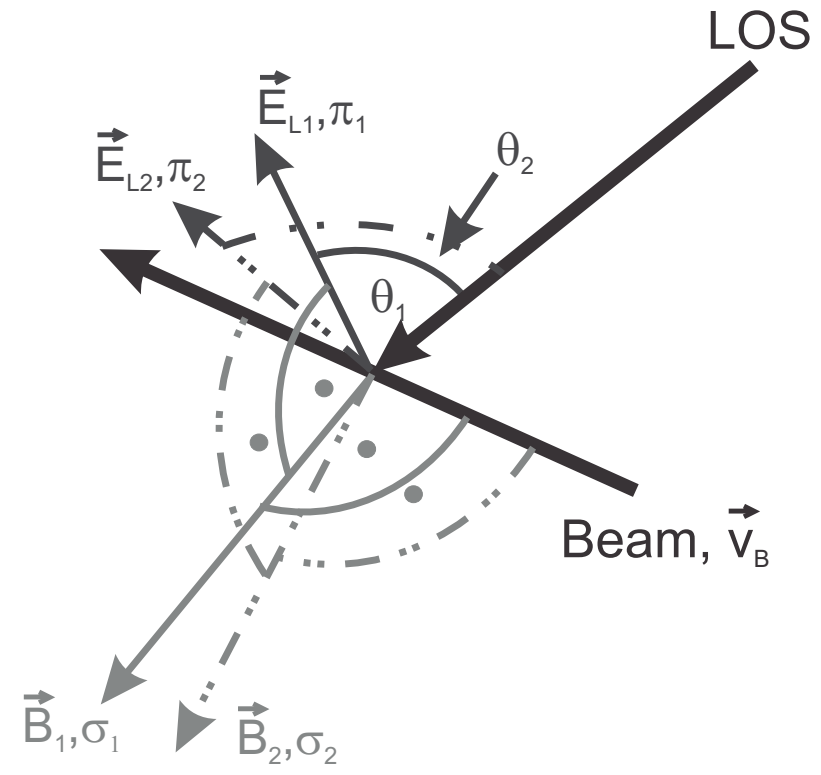

FIG. 12. Schematic representation of sensitivity of $T_{p}^{n s}$ on the magnetic field. LOS: Line of sight, $\vec{E}_{L 1,2}$ : Lorentz field, $\theta_{1,2}$ : observation angle on Lorentz field, $\pi_{1,2}$ and $\sigma_{1,2}$ : polarization characteristic of $\pi$ - and $\sigma$-polarized light, $\vec{v}_{B}$ : velocity of beam particles 